\title{
Effect of Chemical Disorder on Geometrically Frustrated Kagome Lattice
}

\author{
H. $\operatorname{SzYMCZAK}^{a}$, R. $\operatorname{SzYMCZAK}^{a}, \mathrm{M} \cdot \mathrm{BARAN}^{a}$, \\ J. Fink-Finowicki ${ }^{a}$, B. Krzymanska ${ }^{a}$, P. Aleshrevych ${ }^{a}$ \\ AND A. JEZIERSKI ${ }^{b}$ \\ ${ }^{a}$ Institute of Physics, Polish Academy of Sciences \\ al. Lotników 32/46, 02-668 Warsaw, Poland \\ ${ }^{b}$ Institute of Molecular Physics, Polish Academy of Sciences \\ Smoluchowskiego 17, 60-179 Poznań, Poland
}

This paper reviews recent progress in the studies of magnetic dilutions on magnetic properties of two frustrated systems: magnetoplumbite-like $\mathrm{SrGa}_{12-x} \mathrm{Cr}_{x} \mathrm{O}_{19}$ and kagome staircase $\mathrm{Co}_{3} \mathrm{~V}_{2} \mathrm{O}_{8}$ doped with $\mathrm{Mg}$ ions. In the first group of compounds magnetic properties are determined by magnetic defects arising due to doping. In the case of kagome staircase compounds magnetic dilution suppresses effects of crystal field acting on magnetic ions. In particular, the dilution decreases magnetocrystalline anisotropy and anisotropy of magnetization.

PACS numbers: 75.25.+z, 75.30.Gw, 75.30.Kz, 75.50.-

\section{Introduction}

The Heisenberg antiferromagnets on the two-dimensional kagome lattice with only nearest neighbor interactions demonstrate a variety of interesting properties. Such geometrically frustrated magnetic systems could display various exciting low-temperature phenomena such as spin-liquid, spin-glass and spin-ice states [1-3]. Geometrical frustration usually leads to highly degenerate ground state. An experimental fingerprint of this degeneracy is an anomalously low ordering temperature, relatively much lower than would be expected taking into account the strength of the interactions (determined e.g. through the Curie-Weiss temperature). For example in highly frustrated $\mathrm{Y}_{0.5} \mathrm{Ca}_{0.5} \mathrm{BaCo}_{4} \mathrm{O}_{7}$ a Curie-Weiss temperature was determined to be $-2200 \mathrm{~K}$, indicating huge antiferromagnetic interactions, but no magnetic order was observed down to $4 \mathrm{~K}$ [4].

Experimentally, various kagome antiferromagnetic compounds have been studied (see e.g. [5] for references). Theoretically, the $S=1 / 2$ kagome lattice 
has been found to have a spin-liquid ground state [6]. But to date, relatively few studies of the spin-liquid ground state in kagome lattices $[4,7-9]$ have been performed. In order to confirm the existence of the spin-liquid ground state the dynamic magnetic susceptibility is usually measured using inelastic neutron scattering technique.

In real materials one should consider the existence of a magnetic single-ion anisotropy, dipolar interactions, lattice distortions, magnetoelastic coupling as well as magnetic and nonmagnetic doping responsible for the ground states different from that predicted for ideal systems. All these factors are able to change the delicate balance between the competing interactions in frustrated compounds. Each such factor can potentially induce a long-range magnetic order. It is manifested, in particular, through the effects of the substitution of a small amount of magnetic and nonmagnetic impurities on $H-T$ phase diagram of various geometrically frustrated systems. The most of the experimental studies on the chemical disorder caused by impurities in kagome lattices were focused on two groups of materials: the magnetoplumbite-like $\mathrm{SrGa}_{12-x} \mathrm{Cr}_{x} \mathrm{O}_{19}$ and kagome staircase $\mathrm{M}_{3} \mathrm{~V}_{2} \mathrm{O}_{8}$ oxides $(\mathrm{M}=\mathrm{Ni}, \mathrm{Co}, \mathrm{Cu}, \mathrm{Mn})$. In this review we limit ourselves to the effects of substitution studied in these two groups of magnetic oxides.

\section{Kagome antiferromagnet $\mathrm{SrGa}_{12-x} \mathbf{C r}_{x} \mathbf{O}_{19}$}

The geometric frustration in $\mathrm{SrGa}_{12-x} \mathrm{Cr}_{x} \mathrm{O}_{19}(0 \leq x<1)$ arises from a kagome bilayer of the Heisenberg $\mathrm{Cr}^{3+}$ ions with quenched orbital moment and $S=3 / 2$. The disorder in this compound stems from the dilution of the $\mathrm{Cr}$ sublattice by nonmagnetic $\mathrm{Ga}^{3+}$ ions. The magnetic susceptibility measurements (macroscopic, $\chi_{\text {macro }}$, in contrast to local measurements by NMR technique [10]) suggest the occurrence of spin glass state with spin freezing temperature $T_{\mathrm{g}} \approx 2-$ $5 \mathrm{~K}$ [11-15]. All other experimental data confirms existence of a spin-liquid-like ground state $[12,16,17]$. In particular, neutron diffraction experiments indicate the existence of subgroups of spins (singlets, triangles, etc.) [17] and lead to the conclusion that only a fraction of the $\mathrm{Cr}^{3+}$ moments is frozen. The magnetic lattice of $\mathrm{SrGa}_{12-x} \mathrm{Cr}_{x} \mathrm{O}_{19}$ is specific because it consists of kagome bilayers of $\mathrm{Cr}^{3+}$ ions separated by $\mathrm{Cr}-\mathrm{Cr}$ isolated spin pairs. Contribution of these pairs to $\chi_{\text {macro }}$ is described by $\chi_{\text {pair }}$.

At low temperatures $\chi_{\text {macro }}$ deviates from the Curie-Weiss law and crossover to a simple Curie law is observed. This Curie law contribution to the $\chi_{\text {macro }}$ is an effect of the magnetic defects $\left(\chi_{\text {def }}\right)$ arising due to the dilution of the kagome lattice. Finally, according to [10]:

$$
\chi_{\text {macro }}=\chi_{\text {frustr }}+\chi_{\text {pair }}+\chi_{\text {def }} .
$$

The intrinsic (i.e. dilution independent) effect of frustration described by $\chi_{\text {frustr }}$ may be determined directly from the NMR shift of ${ }^{69} \mathrm{Ga}$. These nuclei are coupled to the neighboring $\mathrm{Cr}^{3+}$ ions through a $\mathrm{Ga}-\mathrm{O}-\mathrm{Cr}$ hyperfine bridge. 
It has to be mentioned that the magnetic lattice of $\mathrm{SrGa}_{12-x} \mathrm{Cr}_{x} \mathrm{O}_{19}$ is ideal for occurrence of spin liquid ground state because various interactions (e.g. exchange interactions other than nearest-neighbor interaction, magnetic anisotropy of $\mathrm{Cr}^{3+}$ ions and dipolar anisotropy), usually suppressing spin liquid state, are very small compared to the $\mathrm{Cr}-\mathrm{Cr}$ interactions in this kagome lattice. The results obtained in [10] show that NMR is a very powerful technique allowing to measure directly intrinsic frustration effects through determination of $\chi_{\text {frustr }}$. It was shown that a maximum in $\chi_{\text {frustr }}$ occurs in $\mathrm{SrGa}_{12-x} \mathrm{Cr}_{x} \mathrm{O}_{19}$ at temperatures of about $0.1 \theta$ where $\theta$ is the Curie-Weiss temperature determined using $\chi_{\text {macro }}$ at high-temperature region. This maximum is the signature of the development of short-range antiferromagnetic interactions in the kagome bilayers. The NMR results indicate that the defects in $\mathrm{SrGa}_{12-x} \mathrm{Cr}_{x} \mathrm{O}_{19}$ are associated with a staggered spin response to the vacancies of the kagome bilayers.

The NMR results discussed above are in agreement with neutron-scattering data (reviewed in [12]). It was shown that disorder due to magnetic dilution and geometrical frustration in kagome lattice suppresses the development of magnetic long-range order (even at $T=0$ ). A major obstacle in understanding the frustration in $\mathrm{SrGa}_{12-x} \mathrm{Cr}_{x} \mathrm{O}_{19}$ is the inability to synthesize material with full $\mathrm{Cr}$ occupation. It is in contrast to the situation in second group of materials discussed in this review.

\section{The kagome staircase $\mathrm{Co}_{3-x} \mathrm{Mg}_{x} \mathrm{~V}_{2} \mathrm{O}_{8}$}

Kagome staircase $\mathrm{M}_{3} \mathrm{~V}_{2} \mathrm{O}_{8}$ oxides $(\mathrm{M}=\mathrm{Ni}, \mathrm{Co}, \mathrm{Cu}, \mathrm{Mn})$, have recently attracted considerable attention [18-33]. Their structure is different than standard kagome lattice because kagome layers are not flat but are buckled in and out of the plane. These properties partially relieve the frustration and allow for long-range magnetic ordering at low temperatures. Kagome staircase consists of two inequivalent $\mathrm{M}^{2+}$ sites: the cross-tie sites which form the apices of the nearly equilateral triangles and the spine sites, which form the bases of triangles.

In this review we discuss magnetic properties of $\mathrm{Co}_{3} \mathrm{~V}_{2} \mathrm{O}_{8}$ crystals doped with nonmagnetic $\mathrm{Mg}$ ions. $\mathrm{Co}_{3-3 x} \mathrm{Mg}_{3 x} \mathrm{~V}_{2} \mathrm{O}_{8}$ single crystals were grown with $x=0,0.05$ and $x=0.1$. Neutron diffraction experiments [25, 28, 30] confirm ferromagnetic ordering of pure $\mathrm{Co}_{3} \mathrm{~V}_{2} \mathrm{O}_{8}$ crystals at low temperatures with spin directions along the $a$-axis. According to [28] the cobalt ions on the cross-tie sites deviate from the $\boldsymbol{a}$-axis towards the $\boldsymbol{c}$-axis by an angle $\approx 10^{\circ}$ probably due to frustration. The following phases are observed in $\mathrm{Co}_{3} \mathrm{~V}_{2} \mathrm{O}_{8}$ at $H=0$ :

- paramagnetic $(\mathrm{P})$ above $T_{\mathrm{p}}=11.3 \mathrm{~K}$;

- high temperature incommensurate (HTI) for $8.6 \mathrm{~K} \leq T \leq 11.3 \mathrm{~K}$;

- commensurate antiferromagnetic phase (CAF) for $6.8 \mathrm{~K} \leq T \leq 8.6 \mathrm{~K}$;

- low temperature incommensurate (LTI) for $6.5 \mathrm{~K} \leq T \leq 6.8 \mathrm{~K}$, not observed in magnetic measurements [23]; 
- commensurate antiferromagnetic phase (C) for $6.2 \mathrm{~K} \leq T \leq 6.5 \mathrm{~K}$;

- ferromagnetic phase $(\mathrm{F})$ for $T<6.2 \mathrm{~K}$.

In each of those phases the easy direction of magnetization is parallel to the $\boldsymbol{a}$-axis. The transition $\mathrm{F} \rightarrow \mathrm{C}$ is of the first order.

Figure 1 displays the low temperature dependence of magnetization, $M(T)$, measured in $H=50$ Oe applied parallel to the $\boldsymbol{a}$-axis. It is seen that high level of doping $(x=0.1)$ transforms a very sharp phase transition $\mathrm{F} \rightarrow \mathrm{C}$ into a smeared one probably due to chemical disorder. The observed bifurcation between field-

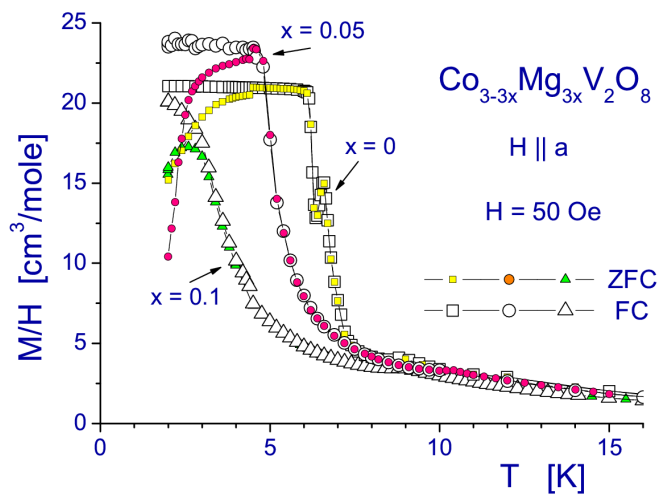

Fig. 1. The low temperature dependence of magnetic susceptibility measured in $H=$ 50 Oe applied along $a$-axis for $\mathrm{Co}_{3-3 x} \mathrm{Mg}_{3 x} \mathrm{~V}_{2} \mathrm{O}_{8}$.

-cooled (FC) and zero-field-cooled (ZFC) magnetization curves may arise due to the presence of the domain structure in ferromagnetic lattice. This bifurcation is completely eliminated at higher fields, when domain structure is expected to be suppressed. Figure 2 presents the $M(H)$ dependences measured in phase $\mathrm{F}$ for

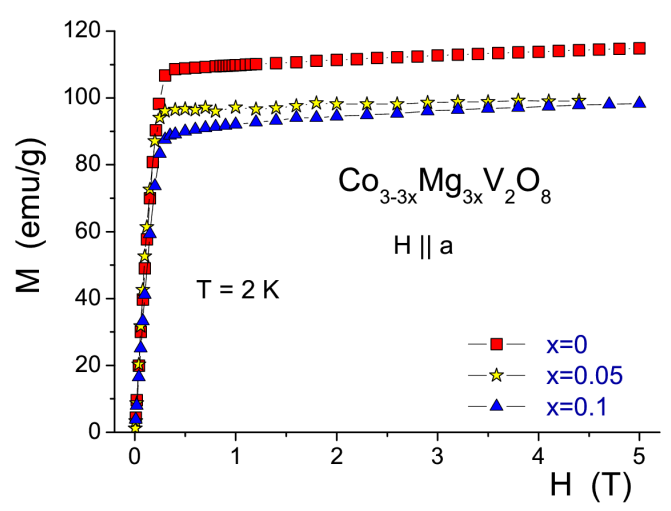

Fig. 2. The $M(H)$ dependences measured at $2 \mathrm{~K}$ in the magnetic field applied along the $a$-axis for $\mathrm{Co}_{3-3 x} \mathrm{Mg}_{3 x} \mathrm{~V}_{2} \mathrm{O}_{8}$. 
various levels of doping and with a magnetic field applied along the $\boldsymbol{a}$-axis. One can notice that the character of magnetization curves does not depend on $x$ - in each case $M(H)$ dependence is typical of a ferromagnetic phase. It confirms that low level doping does not change ferromagnetic character of the ground state and that the direction of the easy axis is parallel to the $\boldsymbol{a}$-axis. For $H \| b$ (Fig. 3) and

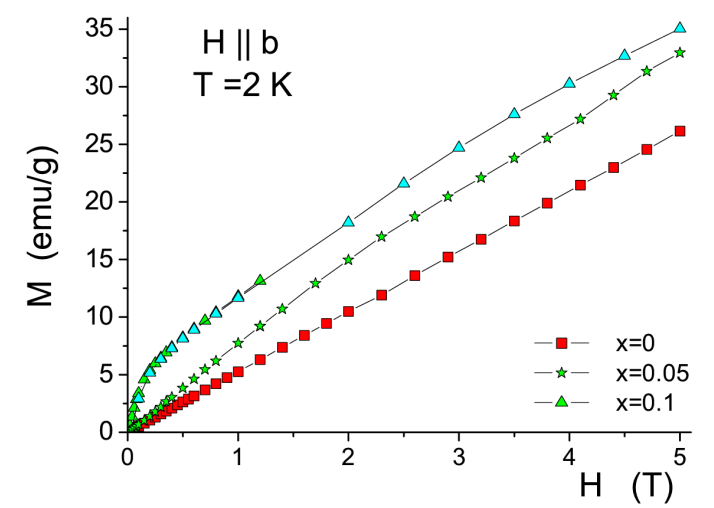

Fig. 3. The $M(H)$ dependences measured at $2 \mathrm{~K}$ in the magnetic field applied along the $b$-axis for $\mathrm{Co}_{3-3 x} \mathrm{Mg}_{3 x} \mathrm{~V}_{2} \mathrm{O}_{8}$.

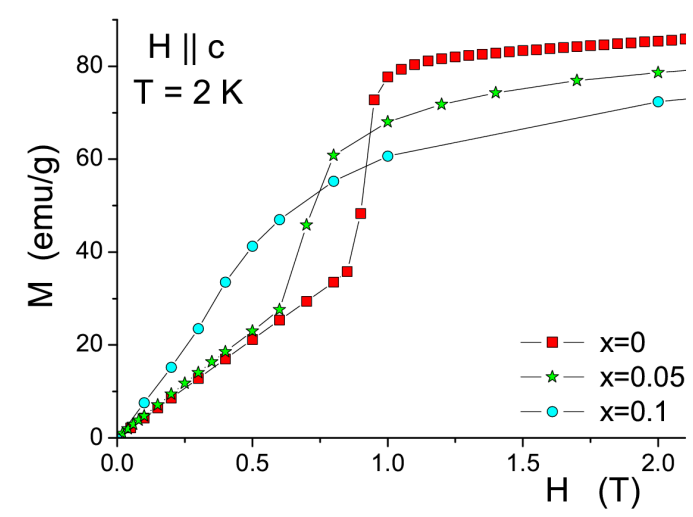

Fig. 4. The $\mathrm{M}(\mathrm{H})$ dependences measured at $2 \mathrm{~K}$ in the magnetic field applied along the $c$-axis for $\mathrm{Co}_{3-3 x} \mathrm{Mg}_{3 x} \mathrm{~V}_{2} \mathrm{O}_{8}$.

for high enough doping $(x=0.1)$ the Dzyaloshinskii-Moriya interactions induce weak ferromagnetic moment along the $\boldsymbol{b}$-axis. Magnetic field applied along the $\boldsymbol{c}$-axis (Fig. 4) induces reorientation of magnetization from the $\boldsymbol{a}$-axis to the $\boldsymbol{c}$-axis. The rotation of magnetization consists of two processes: continuous rotation at low field region and a jump to ferromagnetic-like state. The saturation magnetization in this state is smaller than saturation magnetization observed for $H \| a$. It indicates on strong anisotropy of magnetization in $\mathrm{Co}_{3} \mathrm{~V}_{2} \mathrm{O}_{8}$ crystals 
related to the anisotropy of $g$-factors of $\mathrm{Co}^{2+}$ ions. EPR measurements performed [34] on $\mathrm{Mg}_{2.997} \mathrm{Co}_{0.003} \mathrm{~V}_{2} \mathrm{O}_{8}$ single crystals confirm this concept. The effect of $g$ factors anisotropy is also seen in results of magnetic susceptibility measurements $\chi(T)$ in the paramagnetic phase (see Fig. 5 as an example). The $\chi(T)$ dependences

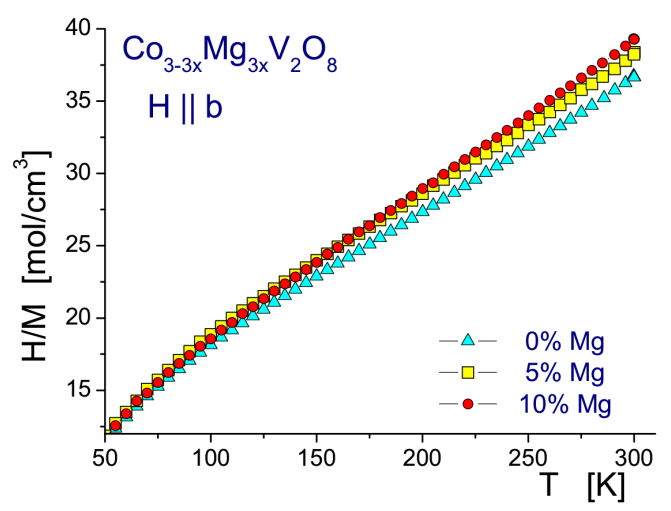

Fig. 5. The temperature dependence of magnetic susceptibility measured in $H=50$ Oe applied along $b$-axis for $\mathrm{Co}_{3-3 x} \mathrm{Mg}_{3 x} \mathrm{~V}_{2} \mathrm{O}_{8}$.

are satisfactorily described in wide temperature range (for $100 \mathrm{~K}<T<300 \mathrm{~K}$ ) by the Curie-Weiss law: $\chi(T)_{i}=C_{i} /\left(T+\theta_{i}\right)$, where $C_{i}=N\left(p_{\text {eff }}\right)_{i}^{2} \mu_{\mathrm{B}}^{2} / 3 k_{\mathrm{B}}(i=a$, $b, c)$. The values of the effective magnetic moment $\left(p_{\text {eff }}\right)_{i}$ are given in Table for $x=0,0.05$ and 0.1 . The effective magnetic moment $p_{\text {eff }}$ decreases with increase

\section{TABLE}

Effective magnetic moments $p_{\text {eff }}\left[\mu_{\mathrm{B}} / \mathrm{Co}\right]$ for $\mathrm{Co}_{3-3 x} \mathrm{Mg}_{3 x} \mathrm{~V}_{2} \mathrm{O}_{8}$ $(x=0,0.05$ and 0.1$)$.

\begin{tabular}{c|c|c|c}
\hline \hline & $x=0$ & $x=0.05$ & $x=0.1$ \\
\hline$H \| a$ & 6.27 & 6.06 & 5.9 \\
$H \| b$ & 5.67 & 5.26 & 5.4 \\
$H \| c$ & 6.10 & 5.90 & 5.6
\end{tabular}

in nonmagnetic doping in agreement with expectations. The determined effective magnetic moments $p_{\text {eff }}$ have the values noticeably larger than the prediction for a spin-only system $\left(3.87 \mu_{\mathrm{B}} / \mathrm{Co}^{2+}\right)$. It is due to the orbital contribution to the overall moment which is not quenched (in such case $p_{\text {eff }}=6.64 \mu_{\mathrm{B}} / \mathrm{Co}^{2+}$ ). The anisotropy of $p_{\text {eff }}$ is in agreement with EPR measurements performed on $\mathrm{Mg}_{2.997} \mathrm{Co}_{0.003} \mathrm{~V}_{2} \mathrm{O}_{8}$ single crystals [34] and suggests that the main contribution to $\chi(T)$ arises due to the $\mathrm{Co}^{2+}$ spine sites. 
All the above reviewed results indicate that nonmagnetic doping decreases mainly the magnetocrystalline anisotropy of kagome lattice and has a weaker effect on other interactions in this frustrated system.

\section{Acknowledgments}

The work was supported in part by the Ministry of Science under the project N202 057 32/1201 and Research Network "Strongly Correlated Fermions".

\section{References}

[1] A.P. Ramirez, in: Handbook on Magnetic Materials, Vol. 15, Ed. K.J.H. Buschow, Elsevier, Amsterdam 2001, p. 423.

[2] S.T. Bramwell, M.J.P. Gingras, Science 294, 1495 (2001).

[3] S.-H. Lee, C. Broholm, W. Ratcliff, G. Gasparovich, Q. Huang, T.H. King, S.-W. Cheong, Nature 418, 856 (2002).

[4] W. Schweika, M. Valldor, P. Lemmens, Phys. Rev. Lett. 98, 067201 (2007).

[5] S. Tanaka, S. Miyashita, J. Phys. Soc. Japan 76, 103001 (2007).

[6] T. Chalker, J.F.G. Eastmond, Phys. Rev. B 46, 14201 (1992).

[7] S.-H. Lee, C. Broholm, M.F. Collins, L. Heller, A.P. Ramirez, C. Kloc, E. Bucher, R.W. Erwin, N. Lacevich, Phys. Rev. B 56, 8091 (1997).

[8] D. Grohol, K. Matan, J.H. Cho, S.-H. Lee, J.W. Lynn, D.G. Nocera, Y.S. Lee, Nature Mater. 4, 323 (2005).

[9] B. Fak, F.C. Coomer, A. Harrison, D. Visser, M.E. Zhitomirsky, Europhys. Lett. 81, 17006 (2008).

[10] L. Limot, P. Mendels, G. Collin, C. Mondelli, B. Ouladdiaf, H. Mutka, N. Blanchard, M. Mekata, Phys. Rev. B 65, 144447 (2002).

[11] A.P. Ramirez, J. Appl. Phys. 70, 5952 (1991).

[12] C. Broholm, G. Aeppli, G.P. Espinosa, A.S. Cooper, J. Appl. Phys. 69, 4968 (1991).

[13] P. Schiffer, A.P. Ramirez, K.N. Franklin, S.-W. Cheng, Phys. Rev. B 77, 2085 (1996).

[14] B. Martinez, F. Sandiumenge, A. Rouco, J. Rodriguez-Carvajal, M. Tovar, M.T. Causa, S. Gali, X. Obradors, Phys. Rev. B 46, 10786 (1992).

[15] B. Martinez, A. Labarta, R. Rodriguez-Sola, X. Obradors, Phys. Rev. B 50, 15779 (1994).

[16] C. Broholm, G. Aeppli, G.P. Espinosa, A.S. Cooper, Phys. Rev. Lett. 65, 3173 (1990).

[17] S.-H. Lee, C. Broholm, G. Aeppli, A.P. Ramirez, T.G. Perring, C.J. Carlile, M. Adams, T.J.L. Jones, B. Hassen, Europhys. Lett. 35, 127 (1996).

[18] N. Rogado, G. Laves, D.A. Huse, A.P. Ramirez, R.J. Cava, Solid State Commun. 124, 229 (2002).

[19] N. Rogado, M.K. Haas, G. Laves, D.A. Huse, A.P. Ramirez, R.J. Cava, J. Phys., Condens. Matter 15, 907 (2003). 
[20] G. Balakrishnan, O.A. Petrenko, M.R. Lees, D.M.K. Paul, J. Phys., Condens. Matter 16, L347 (2004).

[21] G. Lawes, A.B. Harris, T. Kimura, N. Rogado, R.J. Cava, A. Aharony, O. Entin-Wohlman, T. Yildirim, M. Kenzelmann, C. Broholm, A.P. Ramirez, Phys. Rev. Lett. 95, 097205 (2005).

[22] G. Lawes, M. Kenzelmann, N. Rogado, K.H. Kim, G.A. Jorge, R.J. Cava, A. Aharony, O. Entin-Wohlman, A.B. Harris, T. Yildirim, Q.Z. Huang, S. Park, C. Broholm, A.P. Ramirez, Phys. Rev. Lett. 93, 247201 (2004).

[23] R. Szymczak, M. Baran, R. Diduszko, J. Fink-Finowicki, M. Gutowska, A. Szewczyk, H. Szymczak, Phys. Rev. B 73, 094425 (2006).

[24] M. Kenzelmann, A.B. Harris, A. Aharony, O. Entin-Wohlman, T. Yildirim, Q. Huang, S. Park, G. Lawes, C. Broholm, N. Rogado, R.J. Cava, K.H. Kim, G. Jorge, A.P. Ramirez, Phys. Rev. B 74, 014429 (2006).

[25] Y. Chen, J.W. Lynn, Q. Huang, F.M. Woodward, T. Yildirim, G. Lawes, A.P. Ramirez, N. Rogado, R.J. Cava, A. Aharony, O. Entin-Wohlman, A.B. Harris, Phys. Rev. B 74, 014430 (2006).

[26] N. Qureshi, H. Fuess, H. Ehrenberg, T.C. Hansen, C. Ritter, K. Prokes, A. Podlesnyak, G. Schwabe, Phys. Rev. B 74, 212407 (2006).

[27] N. Qureshi, H. Fuess, H. Ehrenberg, T.C. Hansen, C. Ritter, G. Schwabe, Solid State Commun. 142, 169 (2007).

[28] Y. Yasui, Y. Kobayashi, M. Soda, T. Moyoshi, M. Sato, N. Igawa, J. Phys. Soc. Japan 76, 034706 (2007).

[29] A.B. Harris, T. Yildirim, A. Aharony, O. Entin-Wohlman, Phys. Rev. B 73, 184433 (2006).

[30] N.R. Wilson, O.A. Petrenko, L.C. Chapon, Phys. Rev. B 75, 094432 (2007).

[31] N.R. Wilson, O.A. Petrenko, L.C. Chapon, J. Phys., Condens. Matter 19, 145257 (2007).

[32] H. Szymczak, M. Baran, R. Szymczak, S.N. Barilo, G.L. Bychkov, S.V. Shiryaev, Acta Phys. Pol. A 111, 71 (2007).

[33] N. Bellido, Ch. Martin, Ch. Simon, A. Maignan, J. Phys., Condens. Matter 19, 056001 (2007).

[34] P. Aleshkevych, J. Fink-Finowicki, H. Szymczak, Acta Phys. Pol. A 111, 105 (2007). 